\title{
36
}

\section{Advances in Measurement systems integration}

\section{J. Lenner.}

Rockwell Automation

1 Allen-Bradley Drive

Mayfield Heights, Ohio 44124

Ph. 216-646-5114, Fox. 216-646-

5116

Email:Joseph.Lenner@ab.com
F.E. Plonka, Ph.D.

Wayne State University

Dept. of Industrial \& Manufacturing

Engineering

4815 Fourth Street

Detroit, MI 48202

Ph. 313-577-4846

Fax. 313-577-8833

Email:fplonka@mie.eng.wayne.edu

\author{
R. Jaikumar, CMfgT. \\ Wayne State University \\ Dept. of Industrial \& Manufacturing \\ Engineering \\ 4815 Fourth Street \\ Detroit, MI 48202 \\ Ph. 313-577-3821 \\ Fax. 313-577-8833 \\ Email:rkj1@roogna.eng.wayne.edu
}

\begin{abstract}
Two major goals of the Auto Body Consortiun( $A B C)$ are to increase precision of automobile bodies and improve the agility of automotive manufacturing plants. To address the agility issues, $A B C$ has targeted specific processes in automotive manufacturing to facilitate the information integration and exchange among manufacturing entities. These processes, in conjunction with new technology, provide overall quality improvement in the automotive assembly, while reducing the amount of time to develop a new product.

The purpose of this paper is to outline processes for improvement in agility using emerging methods and standards. This paper will focus on the identification of critical parameters and the communication of these parameters throughout the design and manufacturing processes. Key standards will be identified for use and extension.
\end{abstract}

\section{Keywords}

Information Integration, Agile Manufacturing, Auto Body Consortium, Computer Aided Design, Measurement Systems, Standard for the Exchange of Product Model Data (STEP). 


\section{INTRODUCTION}

As companies are adopting strategies to reduce cycle time with leaner organizations, collaborative projects and information integration are frequently mentioned as methods for improving manufacturing productivity and agility. However, many times the communications among organizational functions are impeded by a lack of common tools and methodologies used to identify and communicate the important product features. This leads to a lack of understanding of the items and features that are critical to the overall manufacturing process.

One of the major goals of the Auto Body Consortium (ABC) is to increase the precision of automobile bodies and improve the agility of automotive manufacturing plants. To address these issues, the $\mathrm{ABC}$ has targeted specific processes in automotive manufacturing to facilitate information integration exchange among manufacturing entities. These processes, in conjunction with new technology, provide overall quality improvement in automobile body assembly, while reducing the amount of time to develop a new product.

The Standard for the Exchange of Product Model Data (STEP), ISO $10303^{2}$, was developed to enable effective data exchange between commercial and proprietary product and process design systems. To achieve this communication the standard permits the definition of data models, creation of a database and linkages to programming languages. This paper proposes to use this capability to integrate design and measurement systems. The opportunity exists to enhance communication between individuals performing product design, process design and verification activities. A further opportunity exists to permit intelligent communication between application languages and programmable devices. By extending STEP in this direction, people to people communications among all levels of the industry and the capture of knowledge will be enhanced.

\section{MEASUREMENT SYSTEMS ACTIVITY MODEL}

The overall process for transfer of component design information to the downstream processes for designing and constructing dies, assembly fixtures, part handling equipment, measurement fixtures and measurement procedures requires many different information models. For instance, the sub-assembly instructions for joining of components parts and the "checking" or measurement instructions for critical dimensions in the sub-assemblies, involves the transfer of design information among many different types of design and manufacturing systems. To set the context of the information needs for the various systems, an "activity model", shown in figure 1 , is used to describe the design, manufacturing and measurement operations that are required in each functional area. 


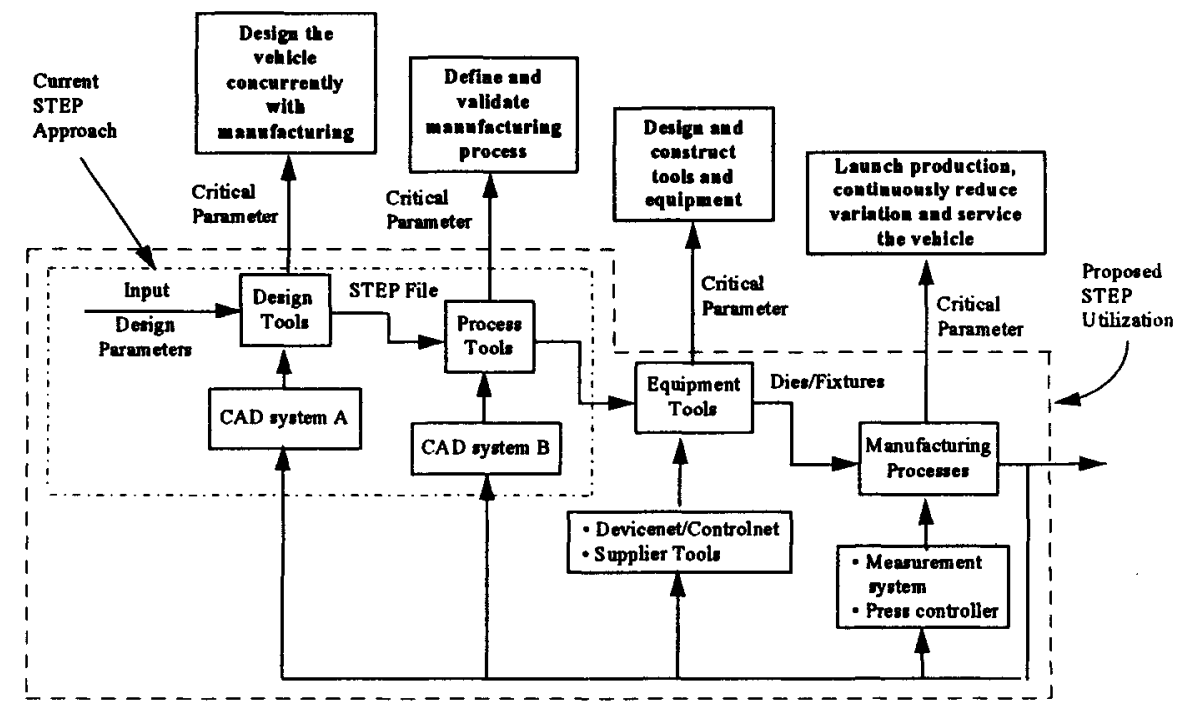

Figure 1 Activity Model

At each stage, the designers will need to identify critical parameters for fabrication and verification. These may be end customer features, assembly features or individual part tooling features. The use of labels in addition to Geometric Dimensioning and Tolerancing (GD \& T) for each critical feature will permit all of the disciplines involved in the processes to effectively interact by supporting a continuity of understanding. These "feature labels" are used to identify critical parameters both for the upstream and the downstream systems and they enable both on-line and off-line measurements to be integrated and communicated to all parties.

In the example shown in Figure 2, a sheet metal assembly is made by joining two sheet metal stamping parts. The product designer has identified the overall width as one of the critical dimensions for assembly to be made from two pieces. The assembly tooling engineers have specified holes and slots to accommodate pins for joining in subsequent assembly operations. The same holes are used to locate the assembly for measurement purposes and identify the relationships between the form and the holes as critical. The die engineers create the form in the first die operation and locate features such as flanges and holes relative to the form. 


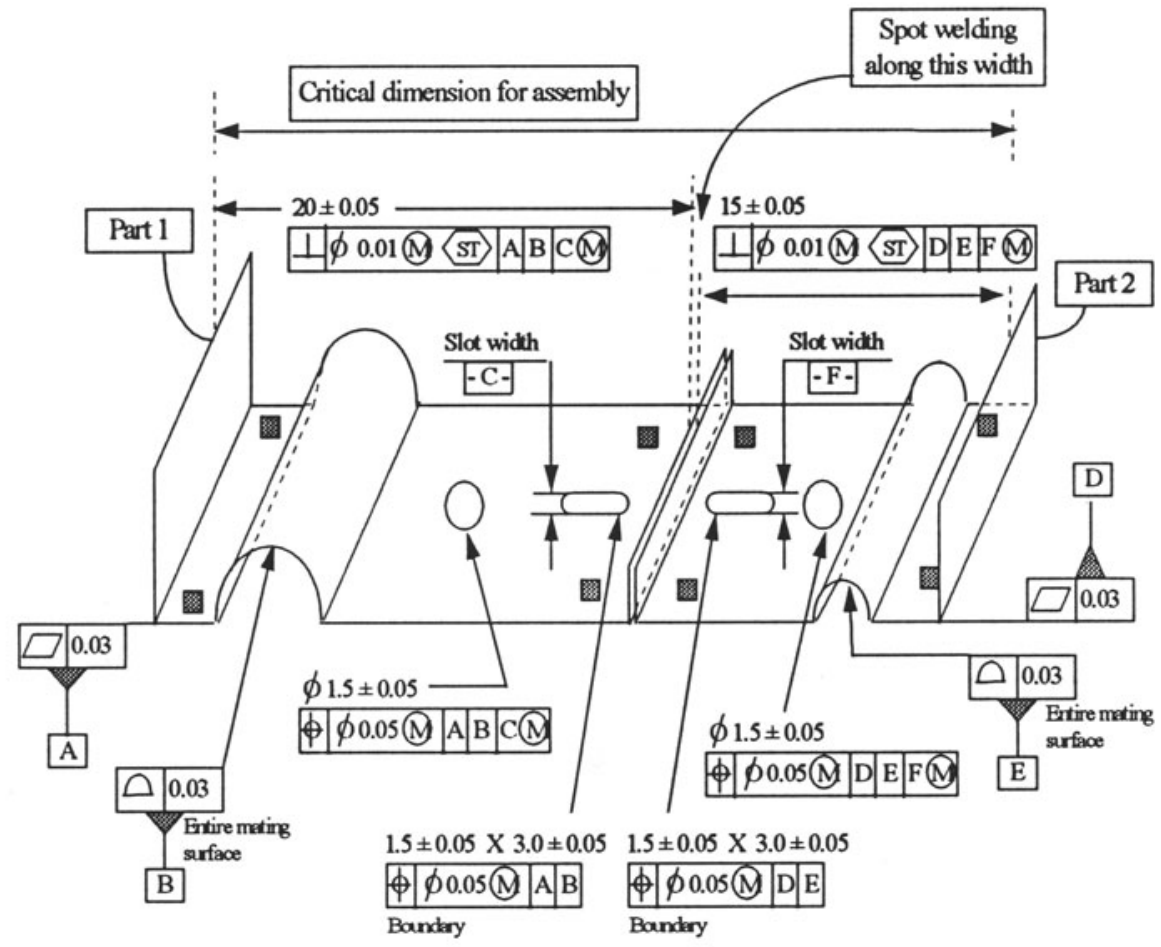

Figure 2 Example of sheet metal stamping and joining operation

The measurement systems that the quality groups will use for the verification at the different process steps must have knowledge of these critical dimensions and their relationships throughout the various process stages. If there is an easily identifiable label associated with each critical feature contained in the drawing, the measurement system will be simplified, minimize unnecessary measurements and contribute to producing quality parts.

The identification of the critical features will also allow the different disciplines to consider the impact that their designs will have on the product. As the product moves through production the consistent identification will enhance the ability of the process engineers to communicate problems, and improvement suggestions effectively to the upstream design personal.

In today's systems there is a gap in our ability to identify and transfer this data to different systems. There are standards which aid in the transfer of the configuration and graphical information, but these standards will need to be enhanced to transfer the identification of critical features. 


\section{CURRENT SYSTEM FOR MEASUREMENTS SYSTEM INFORMATION INTEGRATION}

Many tools for product design today provide the capability to model parts in a 3-D rendering which enables enhanced capabilities for integration of the "component parts" into the total assembly. As these component parts are integrated into a subassembly, the product designer can add additional detail to the parts to specify critical dimensions that must be met in the sub-assembly. These critical dimensions can be utilized to develop a "dimensional measurement" strategy to be used by the downstream measurement systems.

These systems are highly dependent upon manual generation of "paper-based" documentation to define the critical measurements to be made on parts and subassemblies. The documentation is of the form of instructions and sequences of steps to perform the measurements. If new or different measurements are required, "new" instructions need to be developed and communicated.

As the first pilot components and assemblies are built, certain manufacturing considerations may impact the component and assembly design. The ability to relate those manufacturing considerations back to the component or sub-assembly model require annotated changes to be included in the elemental design of the part.

\subsection{Impediments to information integration}

Improving the agility of a manufacturing organization many times requires significant changes in the process as well as the tools used in the process. Technology has enabled many improvements in the tools used in the manufacturing processes. Technology has also enabled more information about local processes to be gathered, stored, and retrieved. However, if this information cannot be clearly shared across functional areas, then only local improvements can be made.

Many of the "information sharing" problems have been addressed by "concurrent" engineering toolsii. This has enabled some of the problems associated with the "waterfall" model of product development to be solved. However, many of these tools simply "import" information from an "upstream" tool into a unique proprietary environment. This requires as many information "import" formats as there are tools. Conversely, the tool typically exports information in only one format unique to that tool environment. Additional problems that may be encountered are:

- Data transformation issues - When measurement systems are used, differences in data formats require a specific re-formatting process. System accuracy issues may impact the translation process.

- Measurement systems integration - Measurement systems are primarily used for local process improvements. When measurement systems are used to feed information concerning the manufacturing process to upstream design functions, the information is not resilient; 
that is, changes in measurement steps and the number of measurements require setting up new procedures.

- Communication of measurement system information - Because of the need to support a distributed and changing supplier base, the communication of new measurement system requirements requires a configuration system to make it available to the "downstream" user measurement systems.

\section{EMERGING SYSTEMS IN INFORMATION SHARING AMONG CAD VENDORS}

Currently in the CAD tool environment, the above problems has been addressed for multiple users of $C A D$ tools, shown in figure 3 , in different design environments such as mechanical, electrical, electronic, etc. by developing standards which enable the "sharing" of information in a common way. An emerging standard, STEP provides a methodology for describing common aspects of a product, which are specific to a particular design discipline $\mathrm{e}^{\mathrm{ii}}$.

STEP is an international standard for the computer-interpretable representation and exchange of product information. The objective of this International Standard is to provide a mechanism that is capable of describing product data throughout the life of a product, independent of any particular computer system.

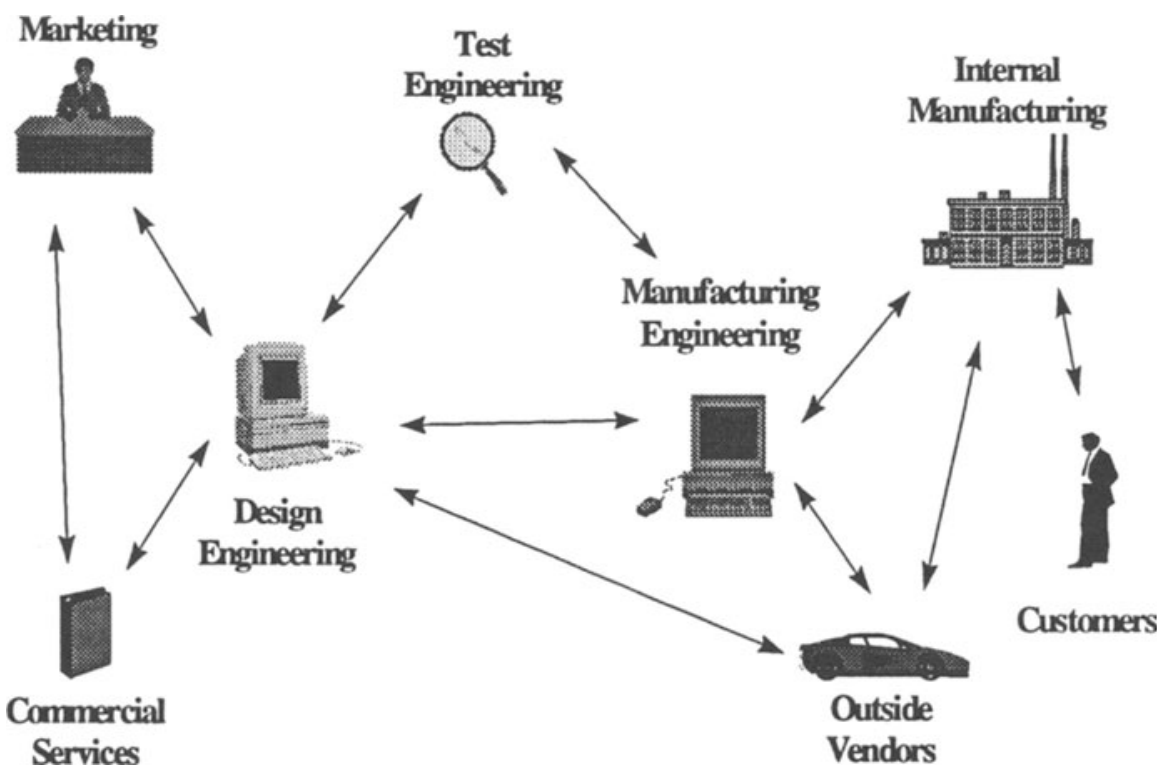

Figure 3 Information sharing among CAD users

STEP is composed of many parts as shown in figure 4. At the core of the standard are the Integrated Resources (parts 40-199, ISO 10303-40 through 199). The 
Integrated Resources define the elements that would be common in many places such as shapes, product structure and geometric dimensioning and tolerancing.

The common language used in all parts of the standard is EXPRESS (part 11). The EXPRESS language specifies data representations in both graphical and textual forms. EXPRESS is a data definition language and as such is not directly executable, but parsers and bindings are available for several of the most commonly used application languages.

The Application Protocols (parts 200-1999) are the parts of the standard that put the basics in the context of the working engineer. The Applications Protocol contains several parts, Scope, Application Resource Model(ARM), Application Integrated Model(AIM), Conformance and Validation, and User Documentation. The scope defines the field of application and bounds the problem. The ARM defines the information requirements in terms an engineer in the specific field would understand. The AIM translates the ARM into EXPRESS for application software developers. The Conformance and Validation sections specify test suites and conformance classes that will be used to compare different applications and implementations by the prospective end users.

ARMs

Application specific models with clearly defined scopes and functional requirements

\section{Resource Models}

Models and Structures involving relationships across those models, that are used to provide required functionality for application

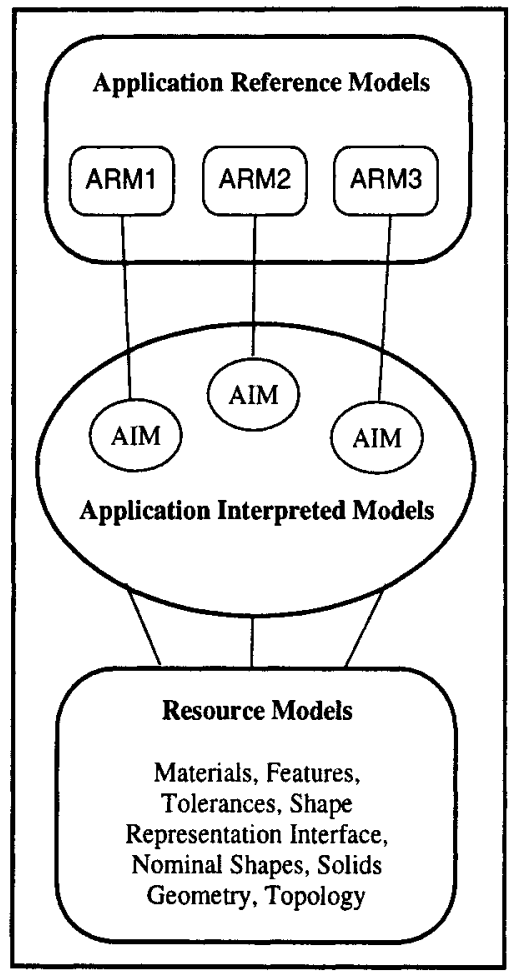

AIMs

Formally describes the 'mapping' between an ARM and the Resource Models 
The actual exchange mechanisms that are in place at this time are for a flat physical file (ISO 10303 part 21) and an Application Programming Interface (API) to databases. The STEP Data Access Interface (SDAI) (SDAI-Part 22) also includes bindings for programming languages such as $\mathrm{C}, \mathrm{C}++$ and Common Object Request Broker Architecture (CORBA) interface description languages (idl's).

While the first versions or implementation of the standard were primarily designed to accommodate exchanges between different CAD vendors of the same discipline(such as mechanical), the methodology also provides the mechanism to define information exchange to different disciplines (such as electrical, electronic, pneumatic, etc.).

\section{CURRENT EMERGING SYSTEMS FOR MEASUREMENTS SYSTEM INFORMATION INTEGRATION}

New systems are emerging to couple the measurement system information to provide access to the design systems. The accuracy information is maintained by keeping the information in a common software environment, as shown in figure 5, or "homogeneous-monolithic" system, or a "math-based" model of the information. Issues with data translation and transformation are minimized because, the "syntax" (the format) and the "semantics" (the meaning) of information are based upon the only one "user interface" in the system.

This common view of the process information enables the upstream/ downstream flow of information to be handled quite efficiently. However, as new tool and/or features are added to the system, the "homogeneous" system must define all of the information that is necessary to describe the product. This creates a tremendous burden for the developers of the "monolithic system" user interface tool environment, because the "user interface" for the design tools need to continually adapt as new requirements are identified. 


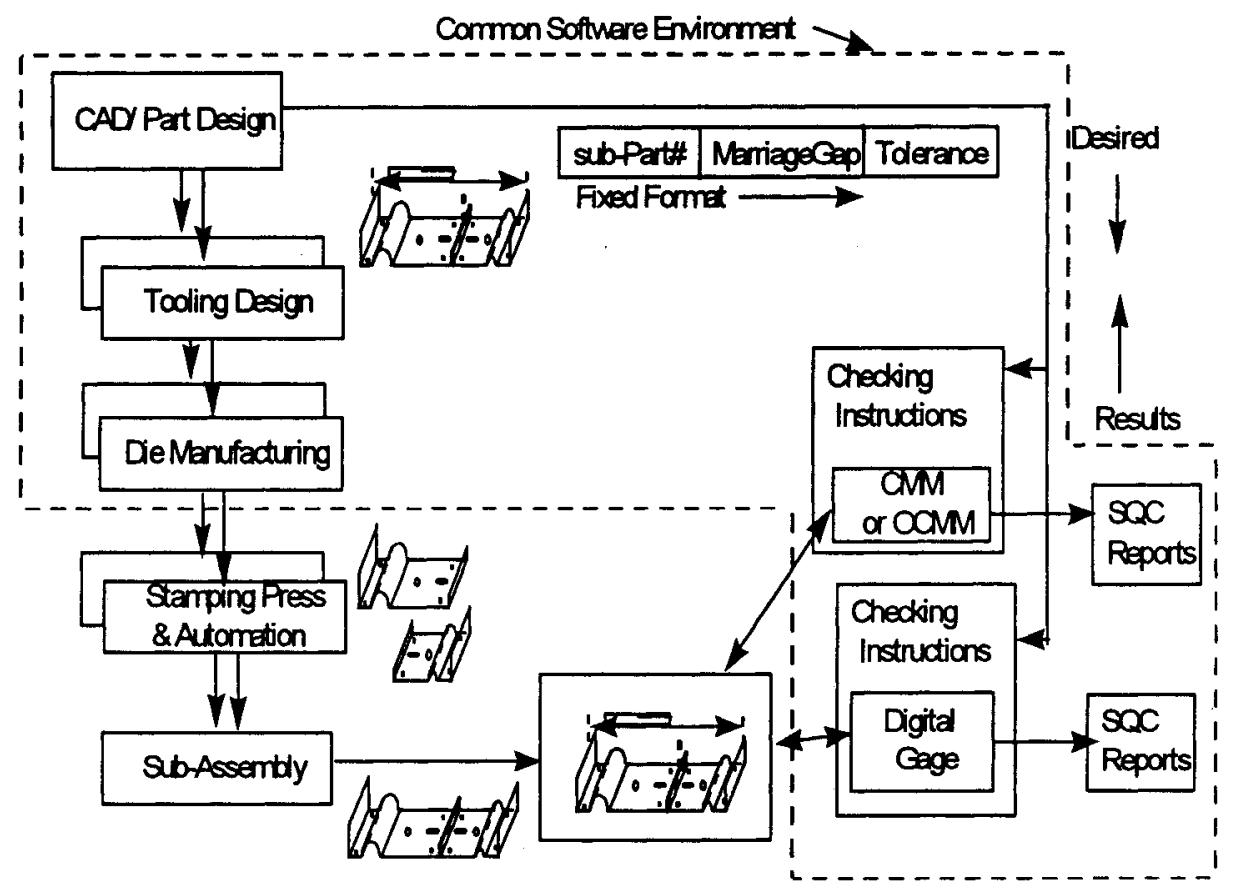

Figure 5 Current emerging systems

\section{PROPOSED SYSTEM FOR MEASUREMENTS SYSTEM INFORMATION INTEGRATION}

This proposal builds upon this concept by first describing standard conventions and guidelines to be used from a particular discipline (such as mechanical design), to the measurement system environment. Inclusion of the information to describe a particular "critical" dimension on a part enables the "downstream" process to "read" the desired information for measurement (see Figure 6 ). 


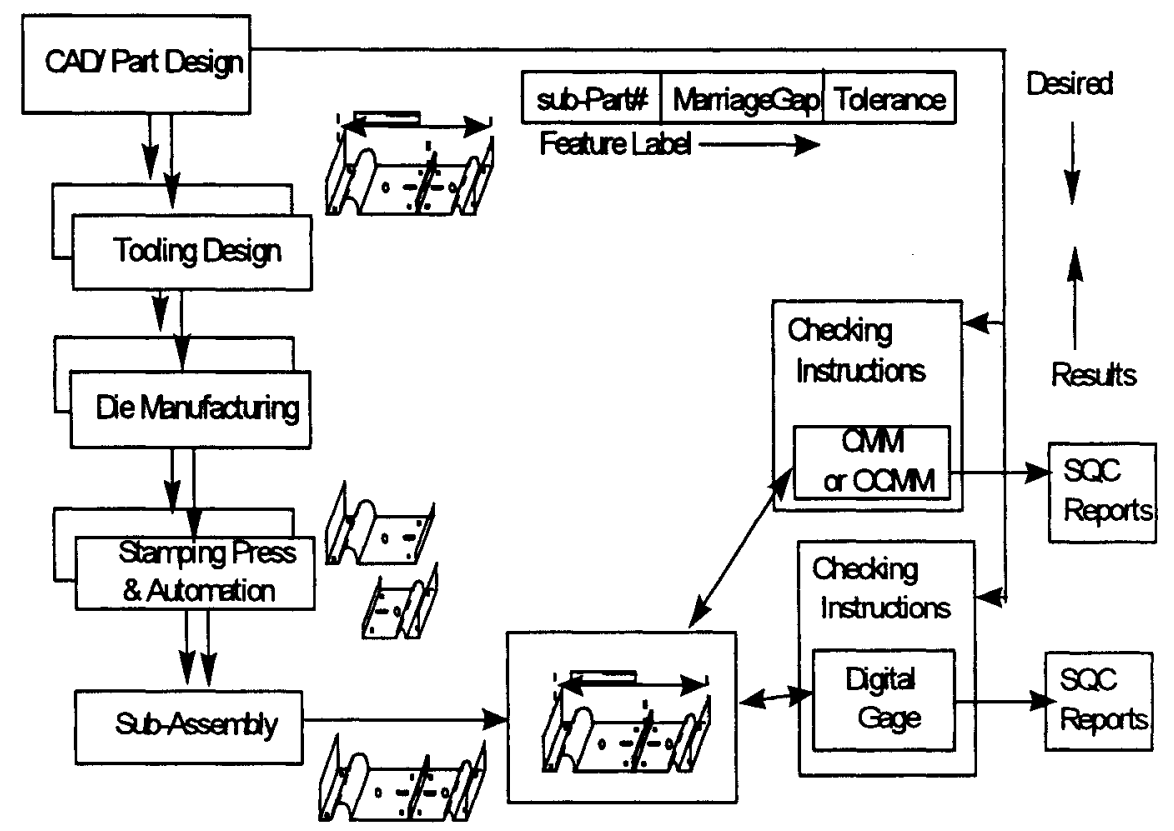

Figure 6 Proposed systems

The proposed system builds upon much of the work completed in the standards and methodologies for the exchange of product information, STEP. This proposal suggest methods for development of guidelines and conventions for the use of those standards to enable the downstream activities to "capture" information from the "upstream" design processes, and to add value to this information by validation of "real measurement" information.

One way that critical features could be identified using STEP is by adding the appropriate identifiers to the entities identifying shape and relationship. Figure $7^{\mathrm{iv}}$, shows one possible way of doing this. The identifiers for "aspect_criticality" and "aspect_relationship_criticality" could be added to the STEP AP-203 Data model as shown. This will allow features and spatial relationships to be identified as critical. 


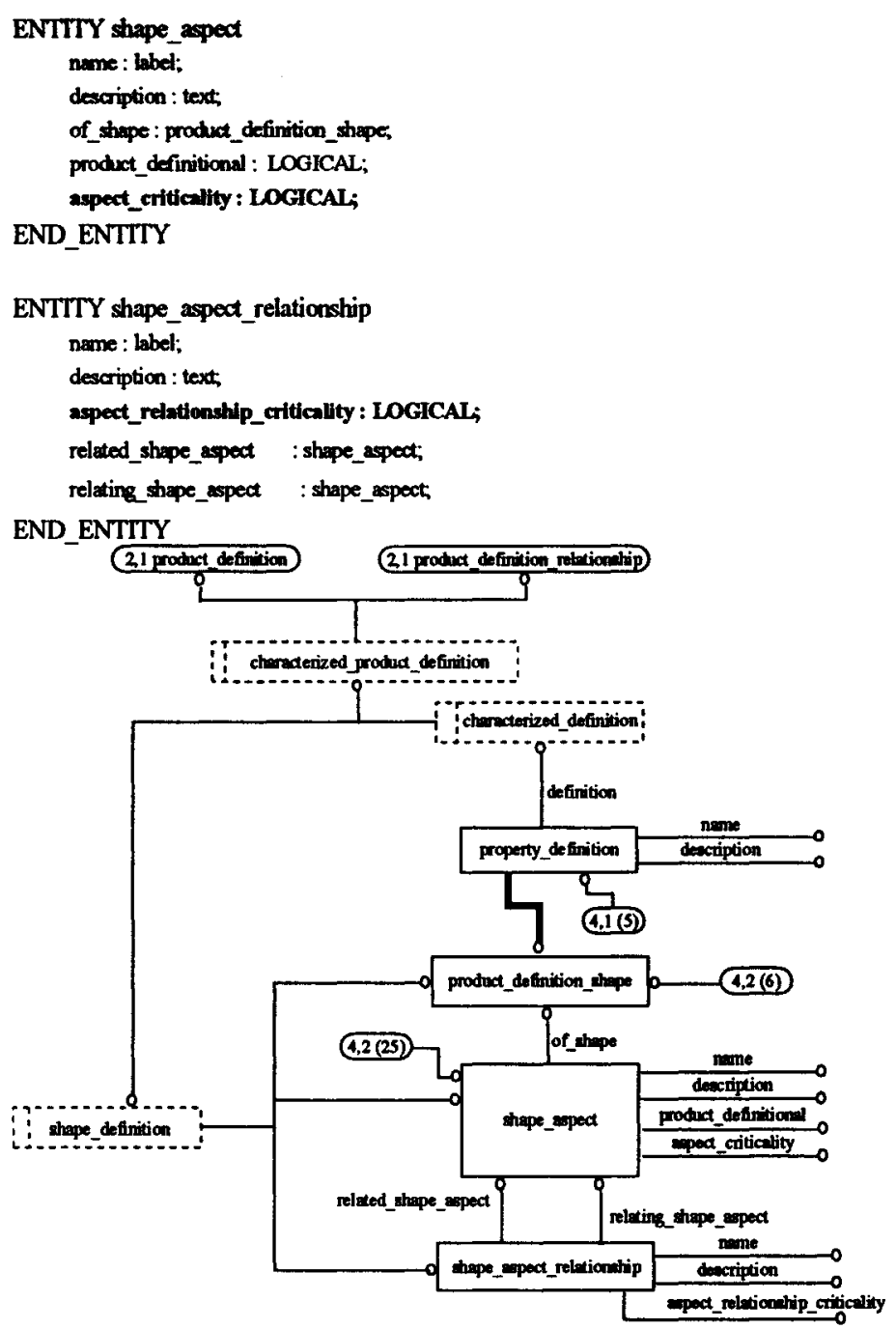

\section{Figure 7 Fragment of AP-203 Data Model}

The ideal place for the identification of critical features to occur is in the Integrated Resources. The inclusion of this identification in the integrated Resources would allow for the propagation of the identification mechanism throughout the appropriate Application Protocols (AP). This inclusion would also facilitate interpretation of differing Mechanical AP's, such as Associative Drafting, 3-D Configuration Controlled Design and a possible future AP for measurement systems.

The development of the "measurement process" or "instructions" are then developed independently of the "design process." Changes to the design can then be "earmarked" for the measurement system (to denote needed changes to the 
instructions). While the sequence of instructions for measurement of the part are not important to the designer, the resultant information may provide information to the manufacturing process engineer in terms of process capability, and information to the Statistical Quality Control (SQC) tools.

\section{SUMMARY}

Information Integration from both upstream/ downstream design and manufacturing activities will become more complex as the capabilities of the tools at each stage of the process improve. The key to controlling and enhancing this process is the utilization of information standards. The clear definitions of the data in the STEP standards will allow information to be shared and clearly understood by both upstream and downstream systems. The sharing and integration of this data eliminates redundant entry of data, speeds the process, increase capability and increases quality.

The use of standard methodologies enables technology improvements to occur at each stage of the design and manufacturing process without requiring all improvements to take place at once. As each process tool improves, the process improvements can be made incrementally. New design and analysis functions can be added without disruption to the manufacturing process provided that the tool supports the appropriate interfaces.

Much as the GD \& $T$ standards have enhanced the ability of the designers to communicate the design information clearly and easily, information standards will continue to facilitate the communications.

The continued enhancement and use of these standards in design and manufacturing tools will offer manufacturers several advantages.

- Common identification of critical features will minimize tooling and measurement errors and expedite the problem solving process when production problems occur.

- Manufacturers will be able to draw from a wider supplier base due to increased information transmission capabilities.

- Manufacturers will be able to choose the best tool at the time, not the best tool that can accept the existing format.

By first adding the ability to identify the critical relationships in the STEP data model the ground work is being laid for the future development of a STEP Application Protocol (AP) for Measurement Systems. The development of a Measurement Systems AP will allow the seamless information integration with of the manufacturing systems, quality systems, product design systems and tooling systems. This level of integration will allow manufactures to realize significant performance and quality improvements while maintaining or decreasing overall costs. 


\title{
8. REFERENCES
}

\author{
' NIST/ATP Near Zero Stamping, 7-95 \\ ii Manufacturing System, July 1996 \\ iii PDES Presentation, 1993 \\ ${ }^{4}$ ISO 10303-203, Configuration Controlled 3-D Designs of Mechanical Parts and \\ Assemblies
}

\section{BIOGRAPHY}

Francis E. Plonka

Frank is a professor in the department of Industrial and Manufacturing Engineering at Wayne State University in Detroit, MI. He presently teaches courses in Operations Management, Manufacturing Information Systems and Senior Design Projects. His research interests include the application of computer methods to designing processes, constructing tooling and nunning manufacturing operations.

Prior to joining the faculty in September 1991, he was Manager, Technical Systems Development on the Advanced Manufacturing Operations Staff of the Chrysler Corporation. In this capacity he was responsible for integrating computers for design, machining, process planning, simulation and factory monitoring into manufacturing operations and training associates in their use.

\section{Raja Jaikumar}

Raja is a graduate research assistant in the department of Industrial and Mauufacturing Enginecring at Wayne State University in Detroit, MI. He is pursuing his Master of Science degree in Manufacturing Engineering with a specialization in Metal Forming and Stanping process engineering. He las worked in the Near Zero Stamping Project of the Auto Body Consortiun, Inc., undertaking research projects involving on-line and off-line measurement stratcgies. 